\title{
Pilgrimage (HajJ) Crowd Management Using AgENT-BASED METHOD
}

\author{
Afnan Alazbah and Bassam Zafar \\ Information Systems Department, King Abdul-Aziz University, Jeddah, KSA
}

\begin{abstract}
Each year, nearly two million Muslim pilgrims from all over the world come to Mecca to perform Hajj, which is the fifth pillar of Islam. The number of pilgrims increases every year, raising concerns regarding how to manage such huge crowds and how to prevent harmful incidents or crowd congestion disasters. The authorities have taken several steps to maintain free-flowing crowds; however, they continue to face difficulties. While numerous studies have been conducted to promote effective and efficient crowd management in Mecca, almost none of them have focused on crowd movements during the Rami AlJamarat ritual, which is when most of the causalities have occurred over the years. None of the studies proposed solutions to directly control the crowd movement without changing the path's current structure. This study proposes a solution to prevent congestion at the Rami Al-Jamarat path without the need to change the structure, and it uses live crowd images to determine proper control signals that are then displayed directly to pilgrims as coloured lights. To demonstrate the results of this solution, a simulation is presented using an agent-based modelling platform.
\end{abstract}

\section{KEYWORDS}

Crowd Management, Hajj, Rami Al-Jamarat Ritual, Congestion, Image Classification, ABMS, CNN Algorithm, AnyLogic.

\section{INTRODUCTION}

Everyday, hundreds of events are held across the world that attract enormous numbers of people, such as festivals, religious gatherings, rock concerts and sporting events [1].The people who gather at such events may belong to different cultures and possess varying educational levels, attitudes and personality traits. A 'crowd' is a group of people who have gathered in a specific place, regardless of their language, nationality, sex or profession [2]. Hajj is an annual religious gathering in which countless people come from all over the world to the holy city of Mecca, Saudi Arabia [3]. These large groups of people move between different religious sites, which has triggered concerns about their safety and security. One of the most important things in the Hajj event is to control and manage the large crowds, ensuring the pilgrims' safety and security and preventing any crowd disasters or stampedes that cause significant loss of life. Therefore, it is critical to detect and reduce crowd congestion as soon as it occurs. This need has motivated us to use the most advanced technologies available in crowd modelling and the simulation domain to reduce the pressure on crowd management forces (police or security) and to warn pilgrims of congestion.

This study focuses on 'conventional' crowds, which are those crowds with members gathered for a specific purpose or to observe a specific event; these crowd members share a common interest. One such scenario that is referred to in this paper is a long crowd of pedestrians (at Mina, near Mecca) flowing in a specific direction and sharing a common goal (The Stoning of the Devil). 
The time pilgrims take to throw stones during the ritual affects the flow of the whole crowd, as part of the crowd stops to throw stones at the pillars, while the remaining crowd in the path keeps moving towards the pillars and other pilgrims continue entering the path. Pilgrim crowds consist of different types of people, including people who are young, old, strong, weak, male and female, and they may walk individually or in groups guided by supervisors. This means they do not move at the same speed, and the time they need to finish throwing the stones varies. These factors play an important role in creating congestion. To prevent congestion, a control mechanism must be applied throughout the path, even at the different entrances, and it will be better if the mechanism is simple, easy to apply, does not require major changes to the path's structure and is accepted by the pilgrims.

In this case study of the Hajj crowd, it is difficult for us to study the pilgrims' behaviours, their speed, density and flow in real time; thus, such a significant and heterogeneous study can be correctly realised using a strong simulation environment. Therefore, we chose agent-based modelling $(\mathrm{ABM})$ as a study tool that simulates and models the behaviours of a huge and heterogeneous system, as well as all their possible interactions and outcomes [4]. Our proposed model should help reduce the number of potential incidents that may occur during crowd movement and, hence, provide a safer environment, specifically in the Jamarat facility, where high congestion may occur (on the day of Eid and days four, five and six of Hajj).

To achieve the above goals, the methodology will be as follows:

1. Collect real-life images from video recordings, which will be used in identifying the crowd's status;

1- Identify the crowd's status using a convolutional neural networks (CNN) classification algorithm, which classifies crowds into three categories: crowded, semi-crowded and normal;

2- $\quad$ Calculate the forces affecting pedestrians and update the agent's parameters before using them to feed the chosen case study;

3- Build a simulation model based on real data that is capable of producing consistent results;

4- $\quad$ Finally, implement the designed model on the ABM platform called Any Logic, which is used for large crowded areas, such as the one chosen in this case study (Al-Jamarat Bridge)

The proposed model consists of two main components. The first component takes images of the moving crowd, and these images are classified using the trained image classifier into three categories: crowded, semi-crowded and normal. The second component consists of three coloured warning lights, and the colour of these lights is based on the results of the classification process: for crowded, the light is red; for semi-crowded, the light is yellow; and for normal, the light is green. This model is spread along all Jamarat hitting paths. Figure 1 presents the model state diagram and shows the classification process and the use of the classification results in determining the correct signal. 


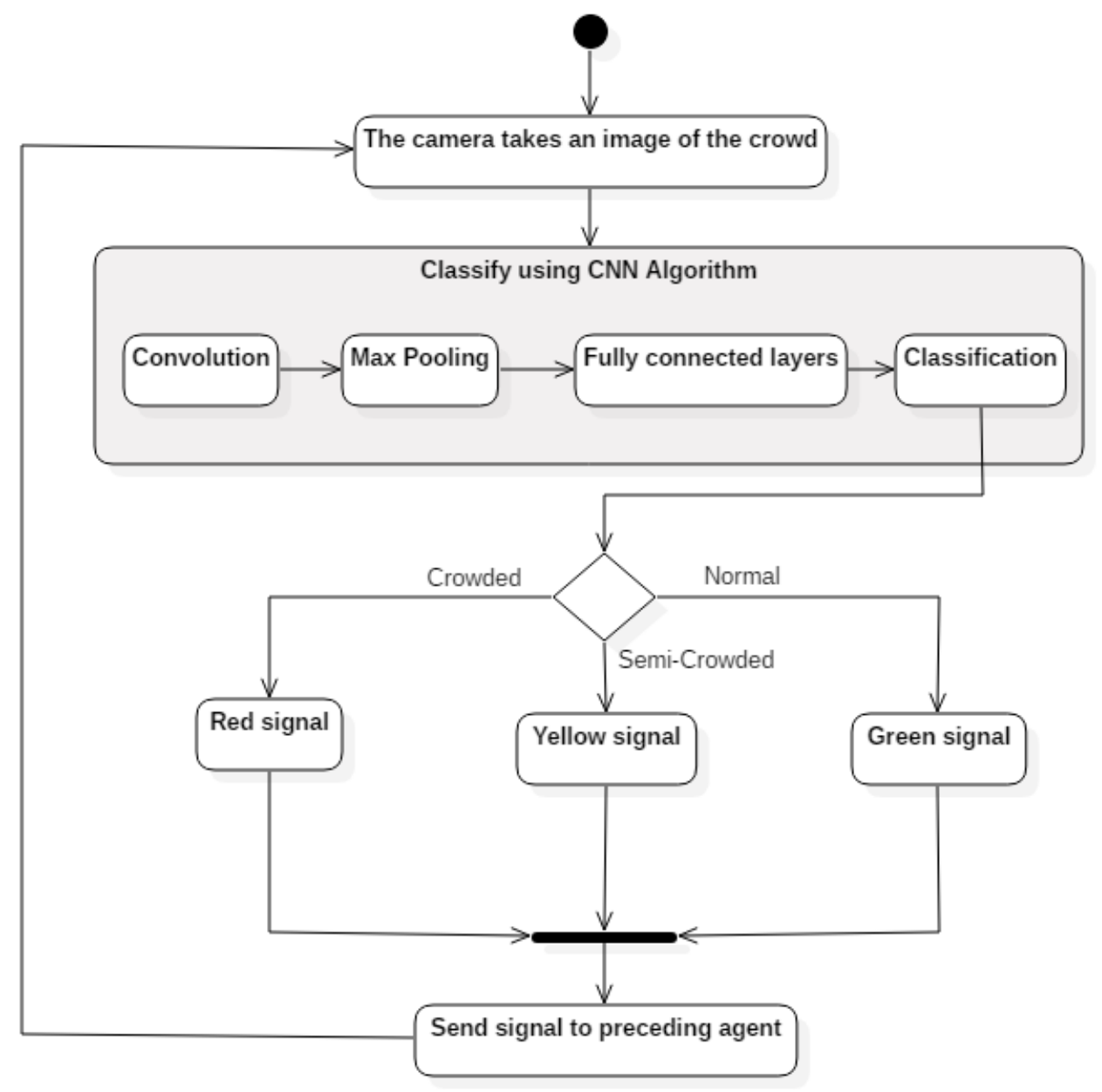

Figure 1. The model state diagram.

\section{RELATED WORK}

In the past few years, crowd simulation and modelling have been attaining significant momentum in investigating pedestrian dynamics. Several simulation methods have been implemented and designed to generate pedestrian behaviour within a crowd. Different behavioural models have been proposed, with various kinds of modelling methods utilised.

Khan and McLeod [5] employed the Agent-Based Modelling and Simulation (ABMS) method to examine the impact of the Al-Masjid-Al-Haram courtyard layout, the Hajj authorities' management preferences regarding the Tawaf crowd's urgent performance based on throughput, satisfaction, health and safety, as well as pilgrim crowd features. Through this method, the performance of micro-level pilgrims was modelled for simulating new crowd performance to produce better safety through reducing casualty numbers. Mulyana and Gunawan [6] undertook a simulation of a Hajj crowd through developing intelligent agents. The simulation was performed for Tawaf, Sa'yee and Jamarat. Furthermore, the suggested system might be employed for training pilgrims before undertaking real tasks. One disadvantage of implementing intelligent agents is the limited number of agents that can be subjected to simulation. However, the number could be expanded with superior computer processing power. Nevertheless, some improvements might be undertaken, including using quantisation with virtual settings, implementing kinetic data structures and using improved mechanisms for detecting collisions. 
In [7], a simulation model was designed to examine the performance for a pedestrian emergency rescue process within large-scale, densely populated settings, such as Mina. The simulation was executed within a cellular automated platform and obtained various behavioural rules and their effects on evacuation process performance, including frequency for updating the selection of exit gates, the path to the exit gate and the exit gate itself. Fayoumi et al. [8] simulated the Rami process using the Steps software tool and evaluated the effect of organising pilgrims into rows near the Jamarat basin. The simulation proposed a linear correlation between the Jamarat basin perimeter and the Rami process throughput. The authors proposed that the overall performance of the Rami process could be improved 25\% through increasing the Jamarat basin perimeter by $20 \%$.

Ilyas [9] suggested the Rami Jamarat ritual model. Net Logo was utilised for developing the model and for simulating the ritual on the basis of user-defined parameters. The parameters included Jamarat's view range, Jamarat's hitting range and time needed for performing Rami. Different Jamarat pillar shapes were evaluated for queuing pilgrims, including circular, elliptical and deformed elliptical. The outcomes demonstrated that the elliptical shape performed best for pilgrim queuing. The results showed the model is still within its initial phases, and several parameters were needed to increase its performance. The model could be enhanced via identifying the pilgrims' significant feelings, including fear, anxiety, confusion and directional focus, which play an important role in crowd dynamics.

Many of the current techniques [5-9] have been carried out to enhance the performance of the Hajj rituals; however,most of them have focused on the Tawaf ritual and the Sa'yee ritual, while few have concentrated on the Rami Al-Jamart ritual.

\section{CONGESTION SCENARIO}

RamiAl-Jamarat (The Stoning of the Devil) is a mandatory Hajj ritual, and it is performed on three days of the Hajj. This ritual takes place on a bridge joining the three pillars: Al-Aqabah, AlWusta and Al-Sughra. Pilgrims are required to move between these three pillars in an ascending order to throw seven pebbles at each of the three pillars[10]. Pilgrims have little time to perform this act. Crowd congestion happens because of the limited time and the huge number of pilgrims who want to perform this ritual. One of the main causes of crowd congestion is that pilgrims must stop to throw the stones, and the flow of the crowd is halted or at least slowed at this particular area of the path while pilgrims keep coming from the preceding areas. Such crowd congestion happens all the time and often becomes extremely dangerous, and people have lost their lives.

Our proposed solution is to act in the early stages of crowd congestion by dividing the whole Jamarat path into a number of areas and to monitor the crowd status in each area; if the number of pilgrims in an area is getting close to a crowd congestion state, we immediately stop the movement of pilgrims in the preceding area to prevent worsening the situation (Figures 2). This method will help in preventing severe congestion and in keeping the crowd flow as close to normal as possible. 


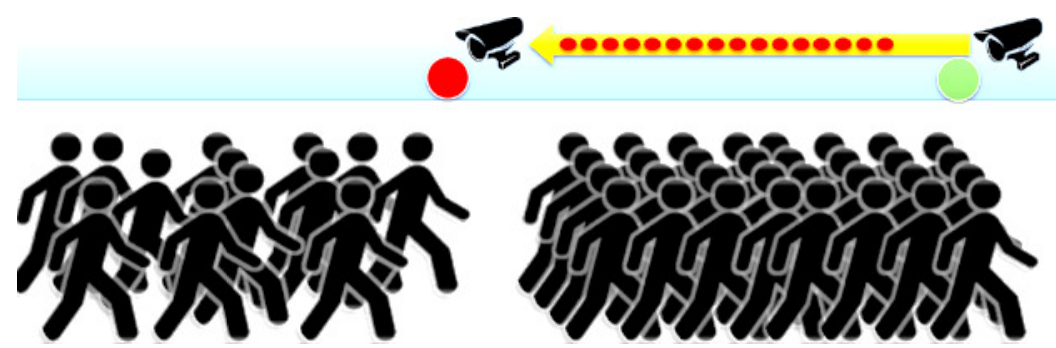

(a)

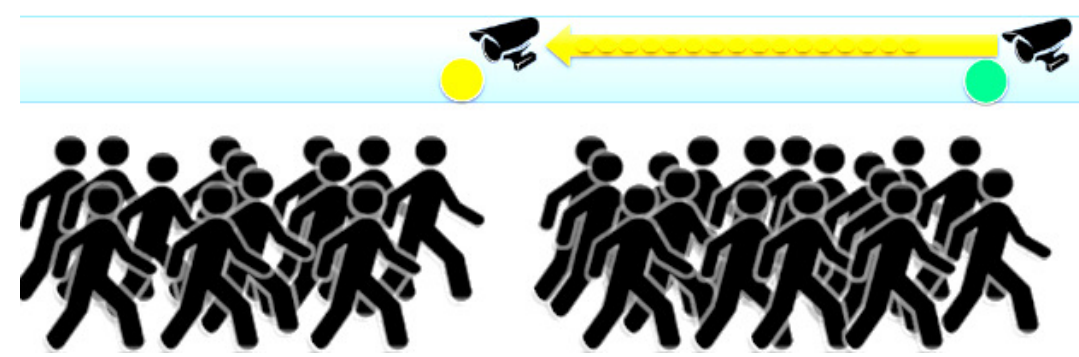

(b)

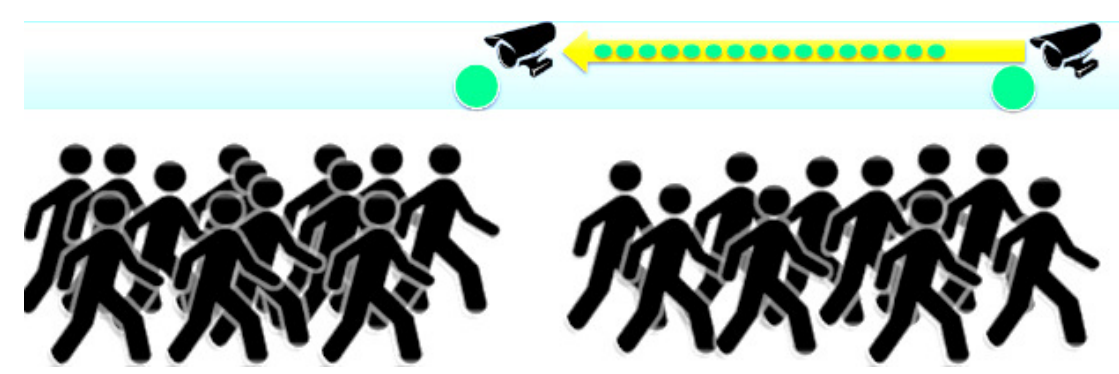

(c)

Figure 2. (a) A monitoring agent at a crowded area shows a green light for the crowd in its area and sends a red signal to the agent at the preceding area. (b) At a semi-crowded area, a green light is shown to the crowd, while a yellow signal is sent to the agent at the preceding area. (c) At a normal area, a green light is shown to the crowd and to the agent at the preceding area.

The model works as follows: The Jamarat hitting path is divided into a number of areas, and a camera and three warning lights (or a three-color warning light) are placed above the moving crowd to cover each area. The camera takes an image of the crowd every few seconds, and then the image is classified into one of the three categories; the image classification determines the colour of the lights in the preceding area (not the area that this camera covers).In the case of a crowded result, a signal is sent to the warning lights in the previous area to become red, yellow for semi-crowded and green for normal. The pedestrian must be informed about the meaning of each light; if the light is red, pedestrians should be warned they are heading to a crowded area and must stop until the light changes; if the light is yellow, it means the pedestrians are heading to a semi-crowded area and must slow their speed to not make it more crowded; and if the light is green, it means that everything is fine and the pedestrian scan move as they wish. This process is continually repeated.

With this method, we prevent congested areas from receiving more pedestrians and becoming more crowded, and it gives the crowd time to become less dense when pedestrians move to other areas.

We adopted image classification instead of counting the number of pilgrims because of its simplicity, and this method is easier to train and achieves high accuracy[11]. A human can tell if the path is crowded without counting the walking pilgrims. Furthermore, we do not need to know 
the exact number of pilgrims; we must simply guess the state of the crowd, and this will be enough for our model to take the correct action. The important thing is to teach the model how to guess with high accuracy during the training process. While we also used the cameras that are already placed on the Jamarat path, we may need to increase the number of these cameras and perhaps change their positioning to get a better view of the crowd. The type of connection between these components depends on the available hardware equipment, and it can be a wired connection or wireless; the decision will be based on the specific location that needs these components.

\section{Image Classification}

\subsection{Image Classification Algorithm}

The software uses supervised training with CNN (Figure 3), and this algorithm has the benefits of neural training and the use of a convolution operation in the image classification process $[12,13,14]$. There are various CNN architectures. The basic components of the CNN algorithm are the convolutional, pooling and fully connected layers [12]. The convolutional layer learns the feature representation of images, and it consists of a number of convolution kernels that are used to compute feature maps. Each layer is followed by a pooling layer to reduce the resolution of the feature maps. After all the convolutional and pooling layers comes the fully connected layers, which contain neurons that are directly connected to other neurons in two adjacent layers.

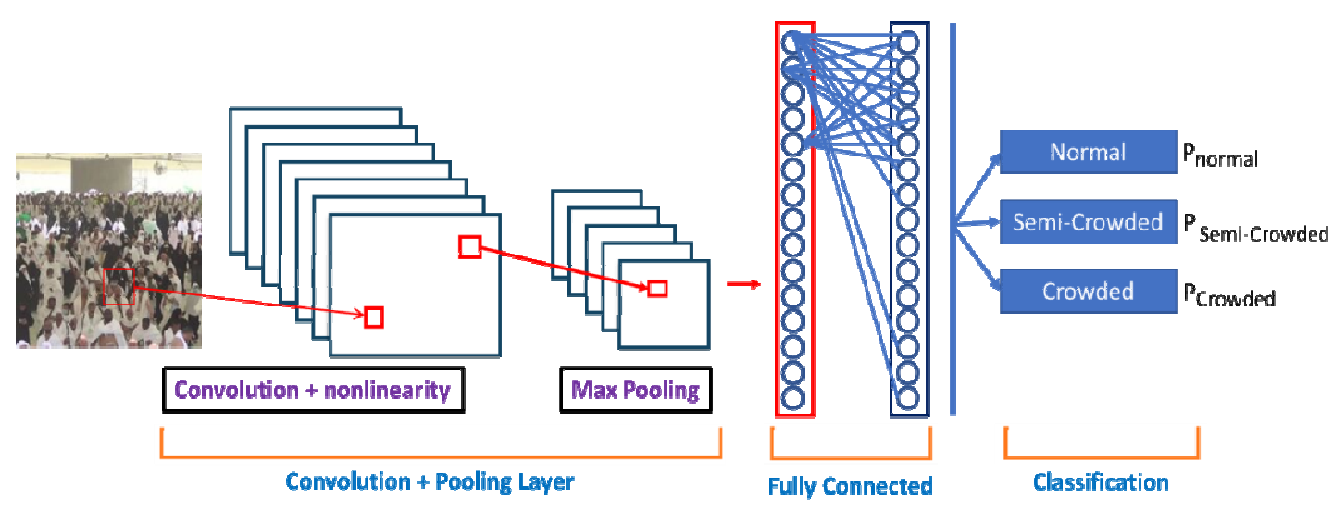

Figure3. The different stages of the CNN algorithm.

\subsection{Dataset}

The classification process must be trained using an efficient dataset that contains a large number of images. Getting the suitable dataset for the moving crowd in the Jamarat path was not easy, as there are insufficient cameras installed along the path, and the existing cameras are not installed at the correct angles; the cameras should be installed facing the crowd from above to cover a straight line of the moving pilgrims. The videos found were from moving cameras that cover the sides of the path. To solve this problem, the researchers were forced to watch several videos to extract suitable images that can be used in the algorithm training process. The main video used for this task featured crowds walking along the Jamarat path in the Hajj season of the Islamic year 1438 (2017).The software 'Free Video to JPG Converter' [15] was used to extract the crowd images. The number of suitable extracted images was 550. These images were used to construct the dataset and were divided manually into three categories according to the crowd state in each image (crowded, semi-crowded and normal; Figure 4).Next, the images were divided into training (420 images) and testing (130) data.To maximise the number of training images in the dataset, several operations were applied to the images, such as flipping, cropping and scaling. 

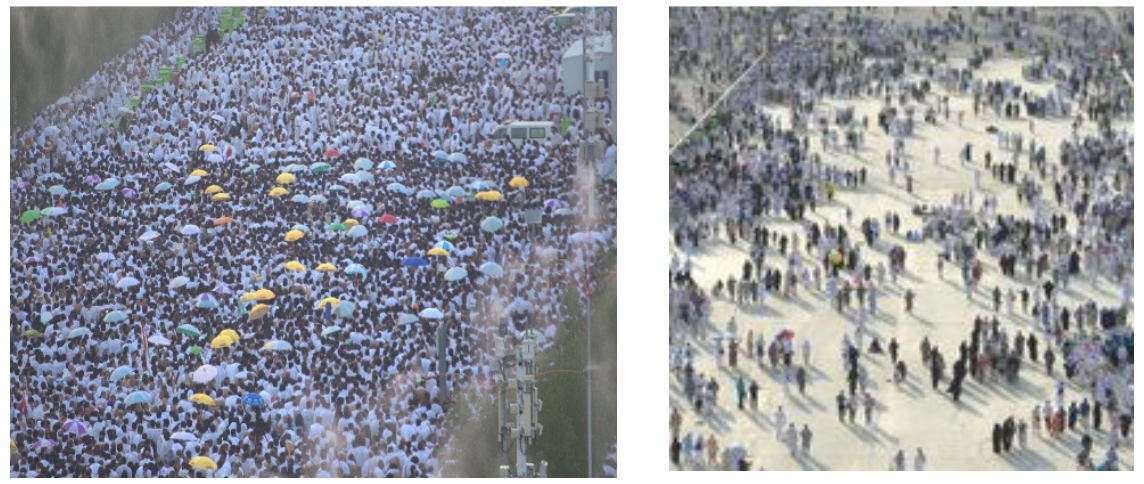

Figure 4. Crowded (left) and normal (right) crowd images.

\subsection{CNN Implementation}

First, we imported the required packages to build the CNN algorithm shown in Figure 3, and we initialised the neural network model as a sequential network because we are using sequential data, Conv2D for the convolution operation, MaxPooling2D for the pooling operation, Flatten for flattening and Dense to perform the full connection of the neural network and all the other required packages. After that, we prepared the images for the training process of the CNN model, and then we initialised the classifier, added the first convolution layer and initialised the input layer and output layer of the fully connected network. At this point, the CNN model was completely built and ready to be compiled. Finally, the data were fit to the model by specifying the training data, the test data and the parameters related to the number of steps in training a neural network.

\subsection{Experiments and Results}

We performed three experiments to get the best accuracy values and to adopt this method into the CNN implementation. The first experiment took many hours to finish, and the results were poor, with the highest accuracy being $36 \%$. Therefore, we improved the algorithm by adding another convolution layer that had 64 filters.

The second experiment took significantly more time than the first; however,the results were good, and the accuracy values started from $80 \%$ at the beginning of training and reached $87 \%$ through improvements. Next, we added a third convolution layer with 128 filters and raised the number of nodes in the input layer of the fully connected layer to 128.

The third experiment took even more time than the previous two; however, the results were better, and the accuracy values started at $85 \%$ in the beginning of the training process and reached $92 \%$. Finally, we added a dropout function that ignores part of the network neurons in the training phase to prevent over fitting in the neural network, and we used 0.2 as a dropout fraction. This experiment was the most successful, with the highest accuracy values reaching $97 \%$. We adopted this CNN implementation, as shown in Figure5. 


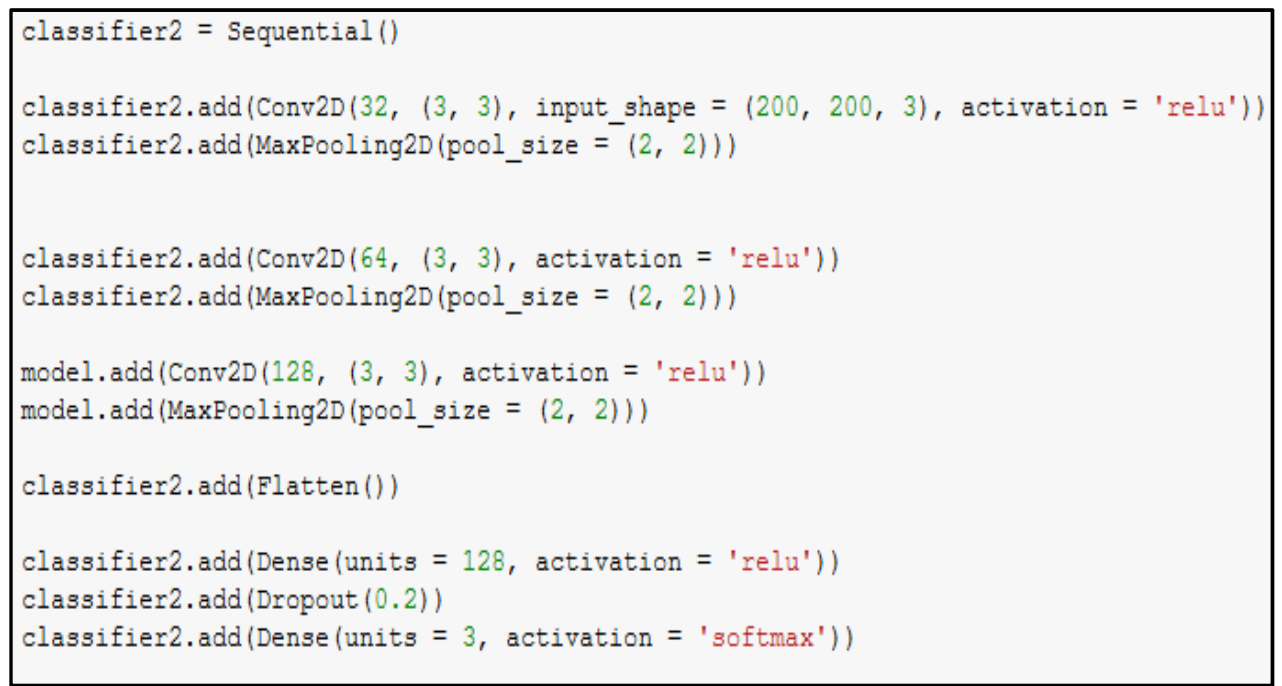

Figure 5. Final implementation of CNN code.

\section{Simulation}

A multi-agent-based simulation has been developed to simulate the crowd along the Jamarat hitting path. To simulate pedestrian behaviour, we needed to select a simulation platform. There are many available agent-based options, and Nikolai and Madey [16] presented a good comparison of these platforms. For our simulation, we chose the Any Logic platform [17], which is a multi-method simulation platform that supports agent-based, general-purpose simulations, system dynamics and process-centric modelling. The modelling language is highly flexible, enabling the capturing of systems' complexity and heterogeneity to any desired level of detail. Any Logic contains the Pedestrian Library, which simulates pedestrian flows in a physical environment, providing the ability to collect statistics on pedestrian density in different areas. When this library is used in a simulation, the pedestrians are simulated as interacting agents with complex behaviour, who can move in continuous space and react to obstacles and other pedestrians. Figure 6 shows the state diagram of the pilgrims' movement along the path. 


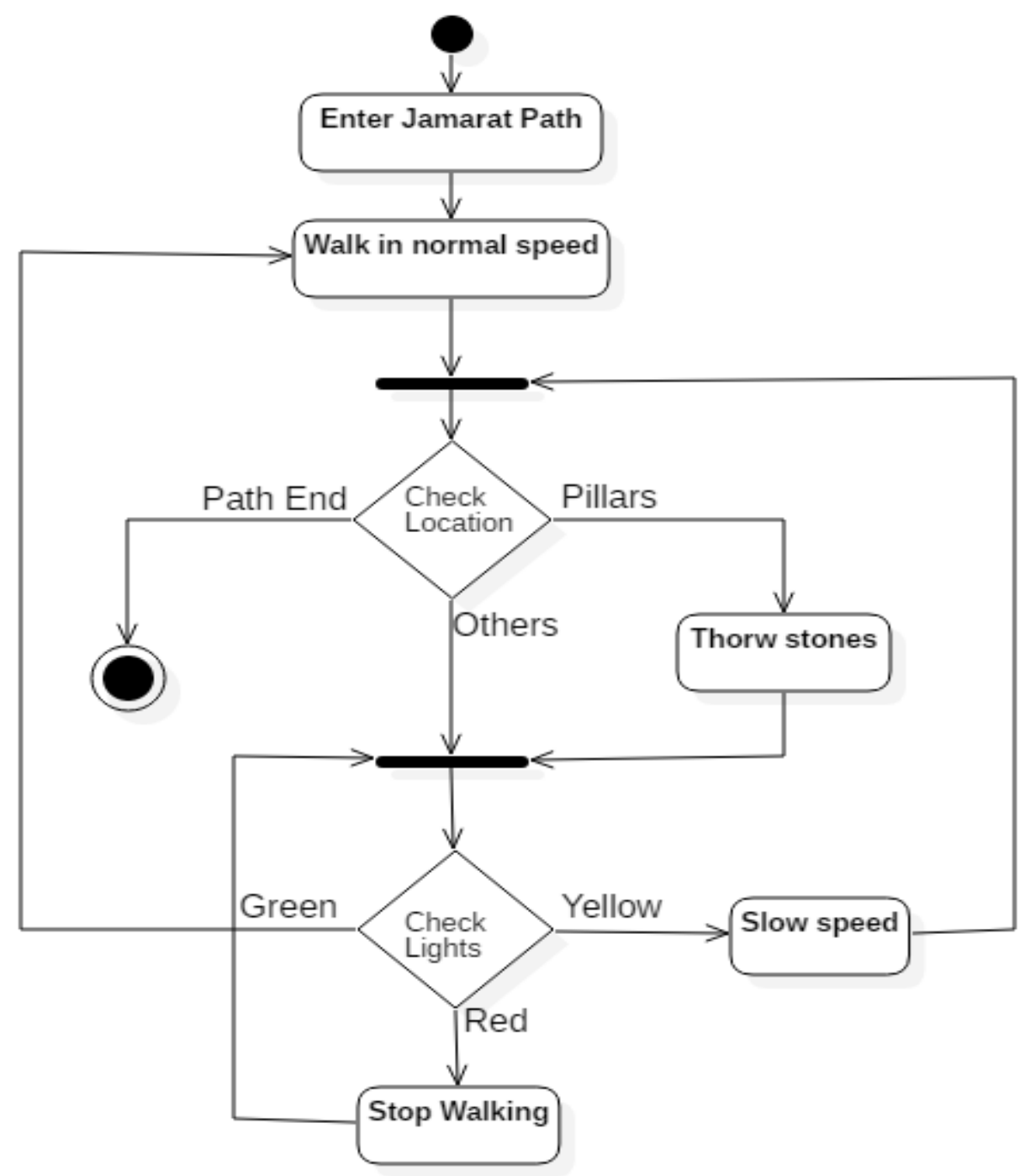

Figure 6. The UML state diagram of the movement of pilgrims alongthe Jamarat path.

\subsection{Simulation Agents}

\subsubsection{Human agents}

Pilgrims are simulated as human agents; each agent has a number of parameters, such as position, speed, size and time needed for stone hitting.

\subsubsection{Force model}

This simulation adopted the continuous force model by Helbing [18]. The Jamarat path is a continuous 2D environment. Agents move freely in this environment, and they represent three types of forces:

1- $\quad$ The internal forces of each agent that make them move to the target.

2- The external forces from other agents in the same path.

3- The external forces from the walls of the path. 
Vector F defines the total force affecting agent movement:

$\vec{F}_{i}=\overrightarrow{\mathrm{F}_{d e s}}+\sum_{j \neq j} \overrightarrow{F_{1 j}}+\overrightarrow{F_{w_{i}}}$

Where Fdes is the internal force that makes the agent move:

$\overrightarrow{\mathrm{F}}_{\mathrm{des}}=\mathrm{m} \cdot \overrightarrow{\mathrm{a}_{\mathrm{des}}}$

$\overrightarrow{\mathrm{F}_{1]}}$ is the force component acting from the centre of agent $\mathrm{Pi}$ to the centre of agent $\mathrm{Pj}$, which is the effect of other close agents:

$\overrightarrow{\mathrm{F}_{1 \mathrm{j}}}= \begin{cases}\left(\overrightarrow{\mathrm{F}_{\mathrm{j}}} \cdot \overrightarrow{\mathrm{b}_{1 \mathrm{j}}}\right) \overrightarrow{\mathrm{b}_{1 \mathrm{j}}} \cdot \overrightarrow{\mathrm{b}_{1 \mathrm{j}}} & , \text { if }\left|\overrightarrow{\mathrm{b}_{1 \mathrm{j}}}\right|<r \\ 0 & \text {, otherwise }\end{cases}$

$\overrightarrow{\mathrm{b}_{1 \mathrm{j}}}$ is the direction vector from $\mathrm{Pj}$ to Pi:

$\overrightarrow{b_{1 j}}=\left(x_{i}-x_{i}\right) i+\left(y_{i}-y_{i}\right) j$

$\overrightarrow{\mathrm{F}_{\mathrm{i}}}$ is the force of the walls affecting agent Pi:

$\overrightarrow{\mathrm{F}_{\mathrm{w}_{\mathrm{i}}}}= \begin{cases}\left(\frac{\mathrm{K}_{\mathrm{w}}}{\mathrm{d}}\right) \overrightarrow{\mathrm{W}_{\mathrm{i}}} & , \text { if } \mathrm{d}>r \\ 0 & \text {, otherwise }\end{cases}$

$\mathrm{K}_{\mathrm{w}}$ is a constant to ensure appropriate force range, $\mathrm{d}$ is the perpendicular distance from the wall to the agent, $\mathrm{r}$ is the influence range of the agent and $\mathrm{Wi}$ is the shortest unit vector from wall to agent Pi.

\section{Updating agent parameters:}

In the simulation, time, location and speed are updated at every simulation time step (Ts), and the new location $(\mathrm{L})$ of the agent at time $\mathrm{t}$ is:

$\overrightarrow{\mathrm{L}_{1}(\mathrm{t})}=\overrightarrow{\mathrm{L}_{1}}(\mathrm{t}-\mathrm{Ts})+\overrightarrow{\mathrm{V}_{1}}(\mathrm{t}-\mathrm{Ts}) * \mathrm{Ts}+\frac{1}{2} * \overrightarrow{\mathrm{a}_{1}}(\mathrm{t}-\mathrm{Ts}) * \mathrm{Ts} * \mathrm{Ts}$

Where $\mathrm{V}(\mathrm{t})$ is the velocity at time $\mathrm{t}$ :

$\overrightarrow{V_{1}(t)}=\overrightarrow{V_{1}}(t-T s)+\overrightarrow{a_{1}}(t-T s) * T s$

Where $\overrightarrow{\mathrm{a}_{1}}$ is the acceleration of the agent $\mathrm{Pi}$ at time $\mathrm{t}$ :

$\overrightarrow{a_{1}}(t)=\frac{\vec{F}_{i}(t)}{m_{i}}$

\subsection{Crowd management agents}

Agents, whose detection range is a rectangular area with length (l) and width (w), manage the crowd movement. These agents are distributed along the whole path, and they communicate with a signal that defines the state of the crowd at each area with one of three colours (red, yellow and green). The proposed model uses cameras and image classification to determine the state of the 
crowd. However, we could not add this part to the simulation; instead, we used a counting process that is available in the Any Logic platform, and we counted the number of pilgrims in each area to determine the state of the crowd.

States of the crowd: The flow rate of people is the number of people passing through a certain area per unit time. To analyse the traffic flow of pedestrians, Fruin [19] developed a level of service standard. Fruin defines six levels of pedestrian flow rates, from level A to level F, with the corresponding density, space, flow rate and speed (Table 1).

Table 1. Fruin's six levels of pedestrian flow rates, with density, space, flow rate and speed

\begin{tabular}{|c|c|c|c|c|}
\hline Level of Service & $\begin{array}{c}\text { Density } \\
\left(\mathbf{p e d} / \mathbf{m}^{\mathbf{2}}\right)\end{array}$ & $\begin{array}{c}\text { Space } \\
\text { (m/ped) }\end{array}$ & $\begin{array}{c}\text { Flow Rate } \\
\text { (ped/min/m) }\end{array}$ & $\begin{array}{c}\text { Av. Speed } \\
(\mathbf{m} / \mathbf{s})\end{array}$ \\
\hline $\mathbf{A}$ & $<0.27$ & $>3.24$ & $<23$ & $>1.3$ \\
$\mathbf{B}$ & 0.43 to 0.31 & 2.32 to 3.24 & 23 to 33 & 1.27 \\
$\mathbf{C}$ & 0.72 to 0.43 & 1.39 to 2.32 & 33 to 49 & 1.22 \\
$\mathbf{D}$ & 1.08 to 0.72 & 0.93 to 1.39 & 49 to 66 & 1.14 \\
$\mathbf{E}$ & 2.17 to 1.08 & 0.46 to 0.93 & 66 to 82 & 0.76 \\
\hline $\mathbf{F}$ & $>2.17$ & $<0.46$ & $>82$ & $<0.76$ \\
\hline
\end{tabular}

Based on these flow rates, we can divide the state of any flow into four states: free flow (A to C), where pedestrians can walk at a comfortable speed; constant flow (D), where the flow is not crowded, yet pedestrians cannot walk at a comfortable speed and are forced to follow the flow speed; crowded flow (E), where pedestrians walk very slowly due to the lack of walking space; and finally, stampede state (F), where pedestrians may lose their balance and fall, perhaps getting injured. The change in the flow state depends on the difference between the flow rate of pedestrians entering the path (Pin) and the flow rate of pedestrians leaving the path(Pout; see Figure 7). 


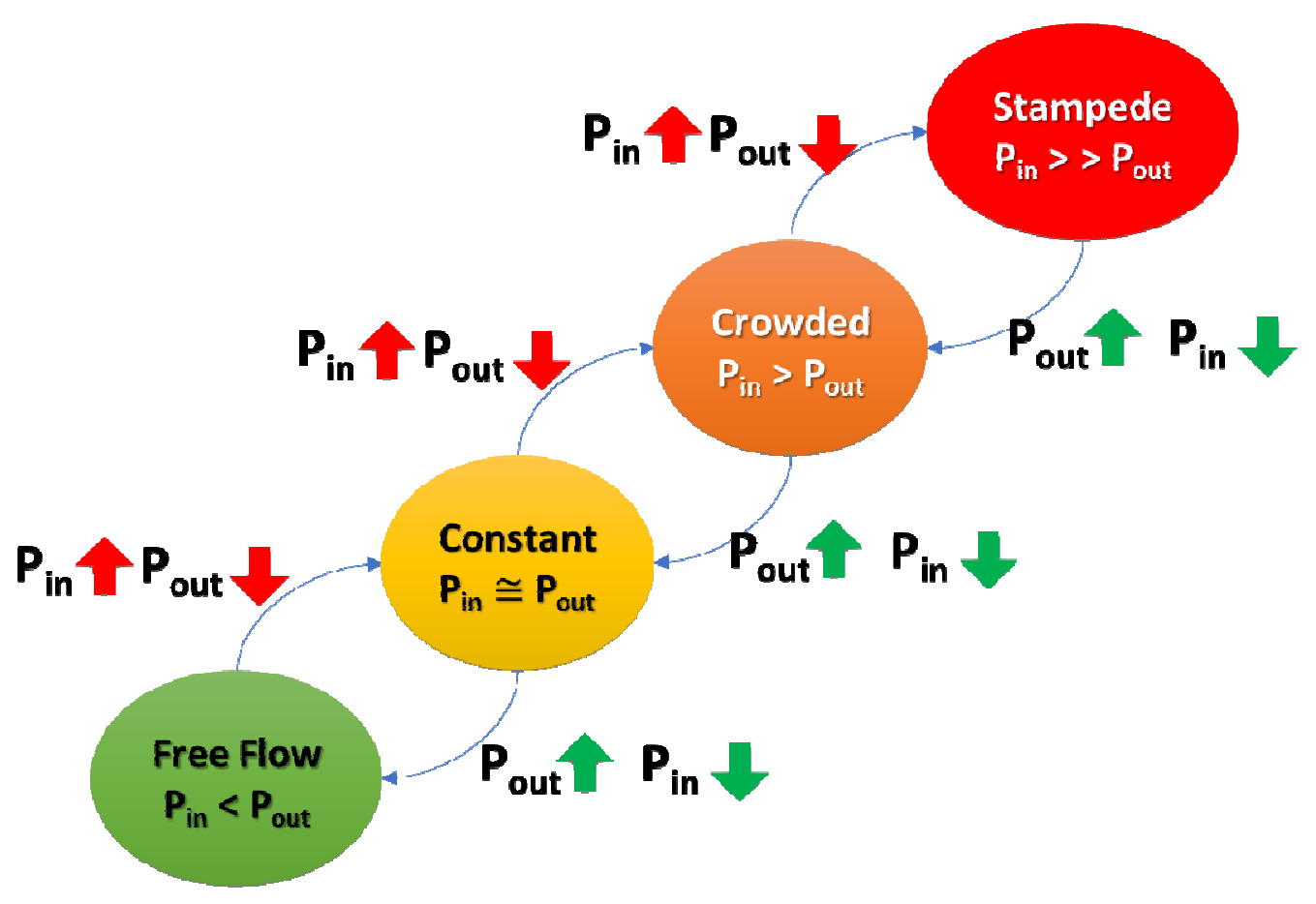

Figure 7. The change in the flow state when the pedestrians entering and leaving rates decrease or increase.

\subsubsection{Crowd simulation}

The simulation is done for a specific area of the path, which consists of the Jamarat pillars, the area before and the area after. The same concept can be applied for the whole path, and congestion in this area is the main reason for congestion in other areas; if we succeed in preventing congestion in this area, congestion will not occur in other areas. We used agents that trigger events to determine the crowd state. We used Java to code the events' details (Figure 8). An agent controls each specific area (e.g. $100 \mathrm{~m}$ long), and the event is triggered three times every minute and counts the number of pilgrims in the area. Based on the results, the state of the crowd in this area can be determined by comparing the results to predefined values for each state (crowded, semi-crowded and normal). Each time the agents ends the suitable signal to the warning lights at the preceding area, the pilgrims will be notified to take the correct action (stop moving, slow down or keep moving). 


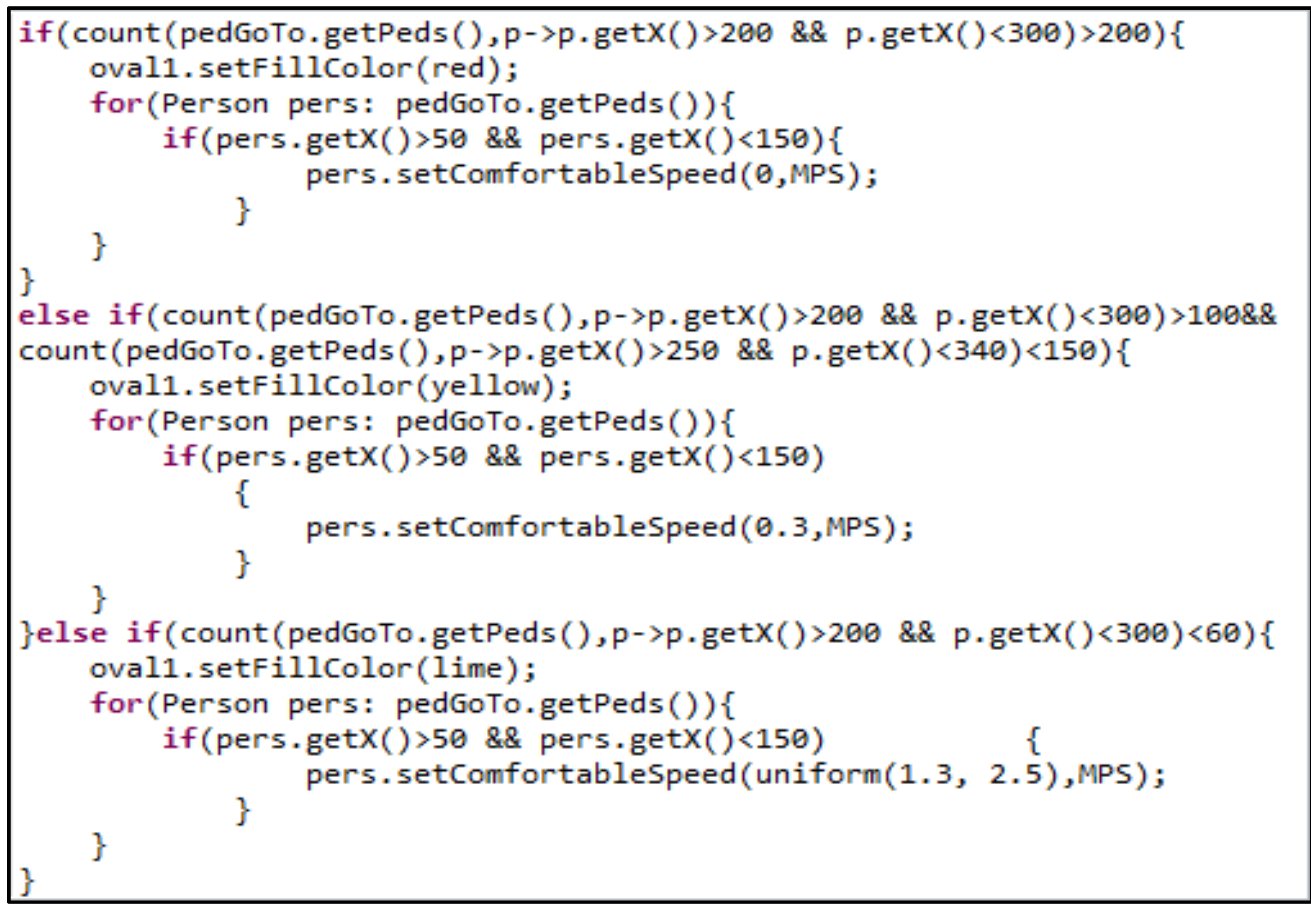

Figure 8. The Java code of the events that monitor and control the crowd movement.

The pilgrims are simulated as moving agents with different sizes and speeds, both as individuals and groups, with the ability to move freely towards the target. These agents stop briefly at the Jamarat pillars to throw stones, and then they continue to the exit. Most agents are designed to follow the directives of the warning lights; however, some agents are designed to not follow these directives.

\subsection{Simulation Results and Evaluation}

In preparing the simulation environment, we drew the path and the pillars, and we also created different variables to monitor the crowd at various locations.

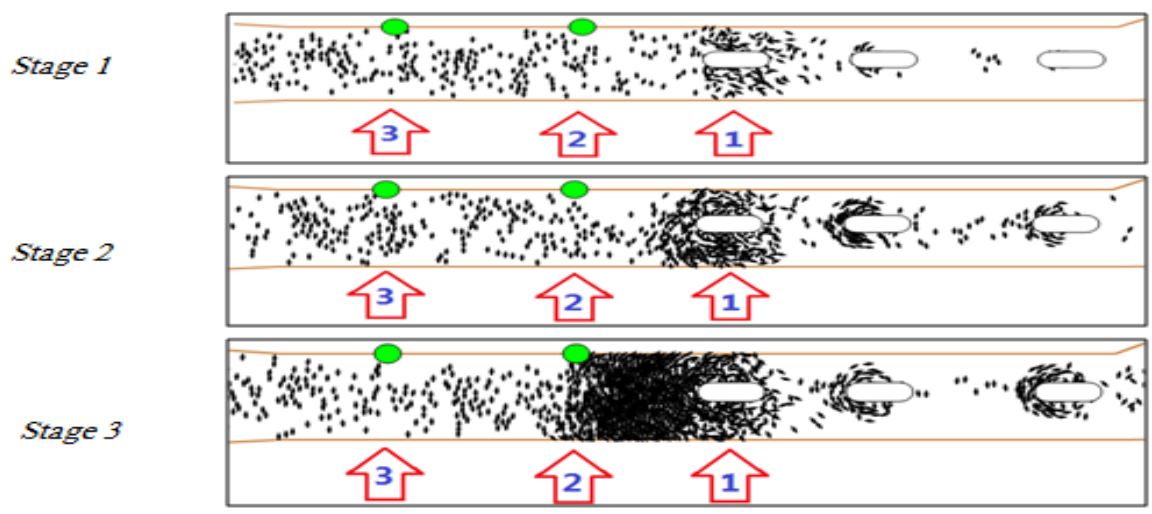

(a) 


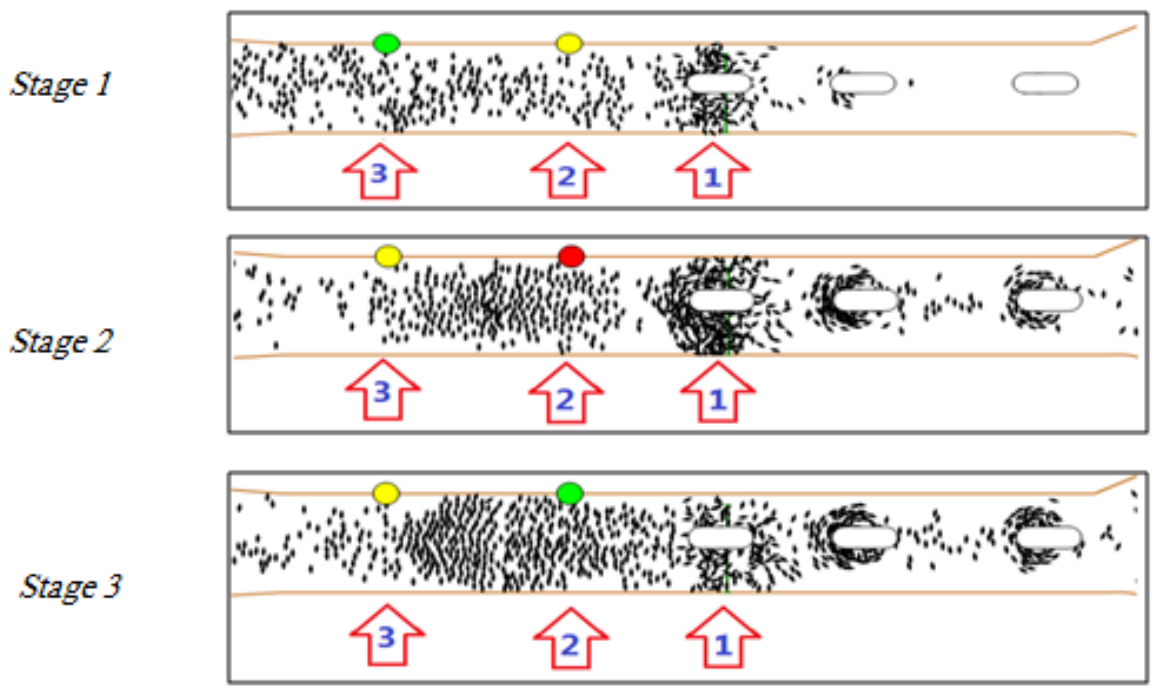

(b)

Figure 9. (a) The simulation without an activating event; (b) the simulation with an activating event.

The stages of the crowd without an activating event (Figure 9 [a]):In stage 1,the flow was normal until the pilgrims reached the Jamarat pillars, where they paused to throw stones and then continued. The number of pilgrims started to rise at location 1, which is the location of the first pillar. In stage 2, more pilgrims were expected to enter this location coming from location 2 , which caused pilgrim congestion at location 1.The numbers of pilgrims continued to rise rapidly, and in real life, this would be a critical situation that threatens pilgrims' lives. In stage 3 , after a few minutes, the situation became catastrophic, as the number of pilgrims at location 1 became extremely large and continued to rise. In such a situation, many people would lose their lives.

The stages of the crowd with an activating event (Figure 9 [b]). In stage 1,the flow was normal until the pilgrims reached the Jamarat pillars, and the number of pilgrims started to rise at location 1 until it became closer to a congestion state. At that moment, the warning light at location 2 was activated with a yellow colour, which directed pilgrims to slow their speed to give more time to the pilgrims at location 1 to move on, which resulted in preventing an increase in the number of pilgrims at location 1 and a change of congestion. In stage 2 , another scenario occurred when the number of pilgrims reached a congestion state at location 1.At that moment, the warning light at location 2 was activated with a red colour, which directed pilgrims to stop moving to give more time to the pilgrims at location 1 , which prevented an increase in the number of pilgrims at location 1 . This led to ending the congestion situation at location 1 and to preventing a more severe congestion. In stage 3 , the situation after the previous scenario is seen, and the warning light at location 2 became green, which meant that pilgrims at location 2 could continue their movement. When pilgrims stopped at location 2, their numbers rose rapidly, which activated the warning light at location 3 with a yellow colour to prevent congestion.

\section{Comparing the number of pilgrims entering with the pilgrims leaving.}

The effect of using the proposed flow model of pilgrims is seen in the difference between the number of pilgrims entering any area of the path and the number of pilgrims leaving this area. Without using the model, there is a significant difference between the pilgrims entering and leaving the area, and the number of those entering is much higher than those leaving the entire 
time (Figure 10 [a]). However, when the model is used, the difference is not significant, and sometimes the leaving rate is higher than the entering rate (Figure $10[\mathrm{~b}]$ ).

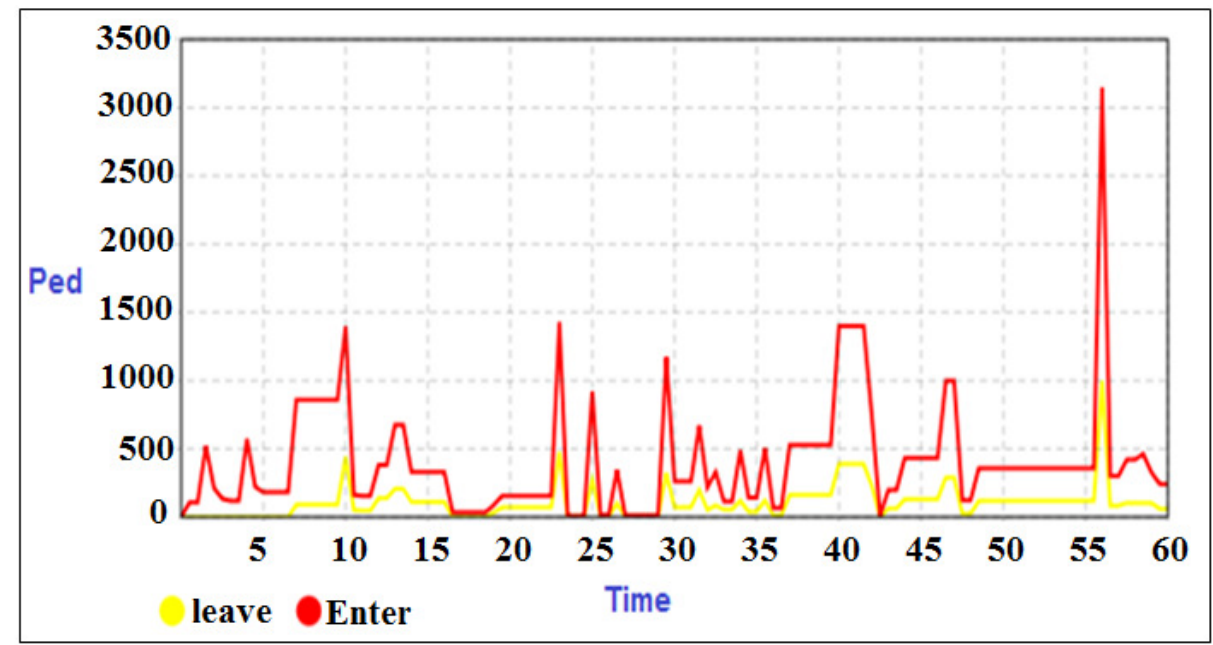

(a)

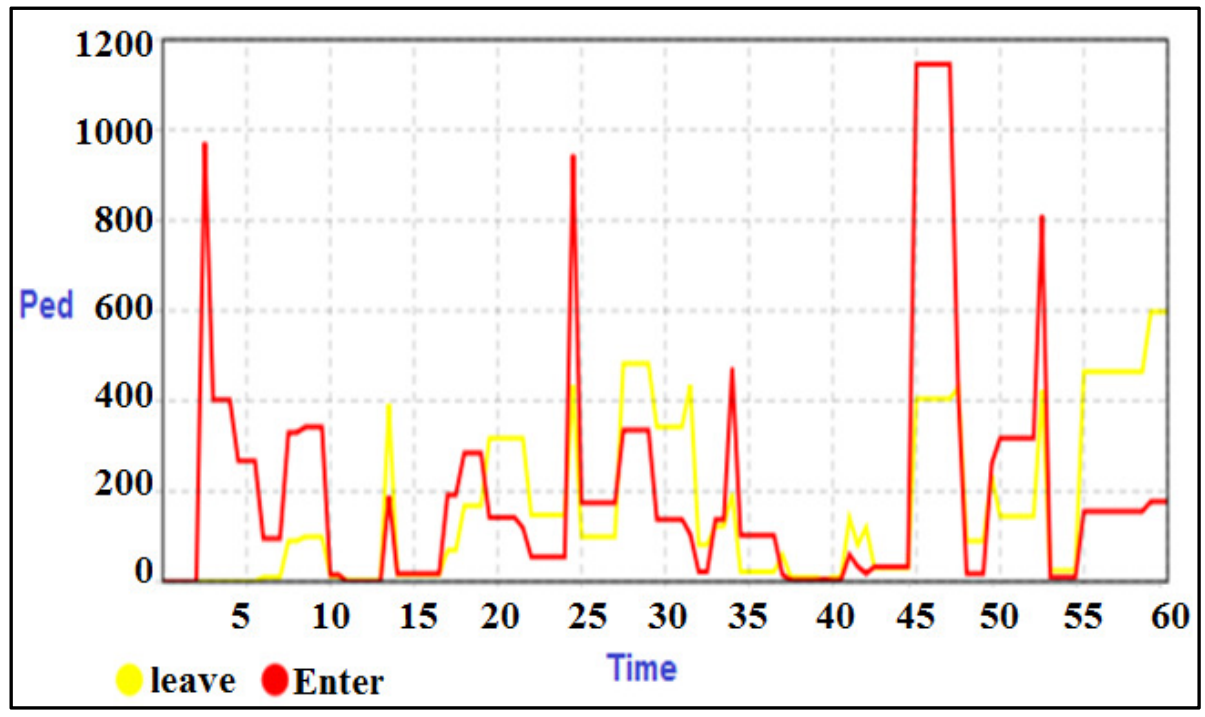

(b)

Figure 10. (a) Comparing the number of pilgrims entering a specific area with the number of pilgrims leaving this area without applying the proposed model and (b) with applying the proposed model.

\section{Density at a specific area:}

The next plots show the density (ped/m2) at a specific area of the Jamarat path in two situations. The first situation is when we do not apply the proposed model (Figure 11[a]). We know from Fruin's [19] level of service that normal flow has a density less than $0.8 \mathrm{ped} / \mathrm{m} 2$; thus, the density has an extremely high value that reaches a stampede state without applying the proposed model. However, when applying the proposed model, the pedestrian density remains in the normal flow state (Figure 11[b]). 


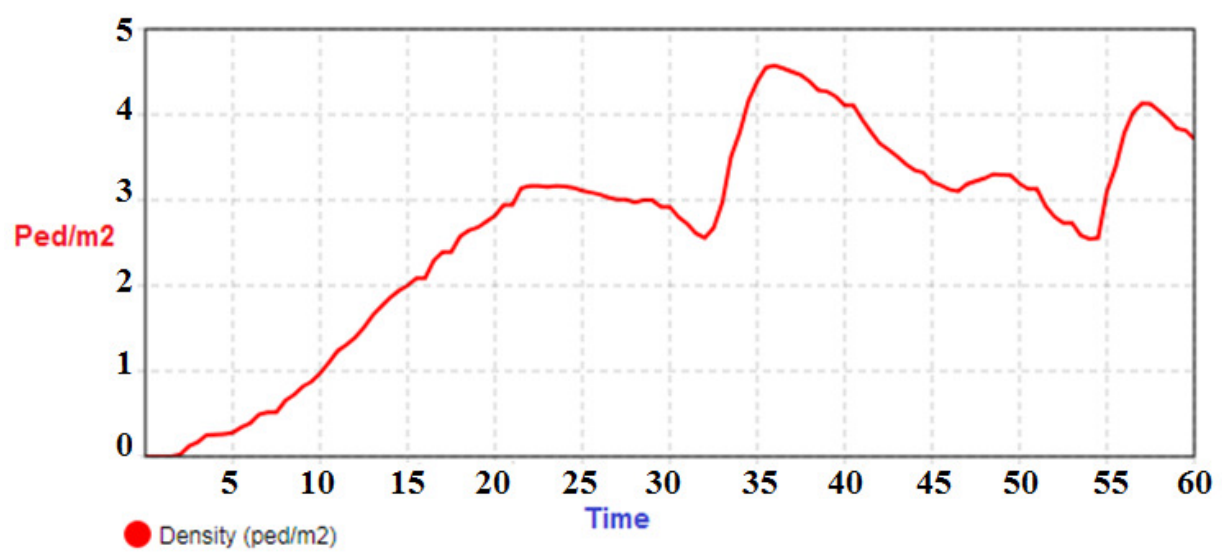

(a)

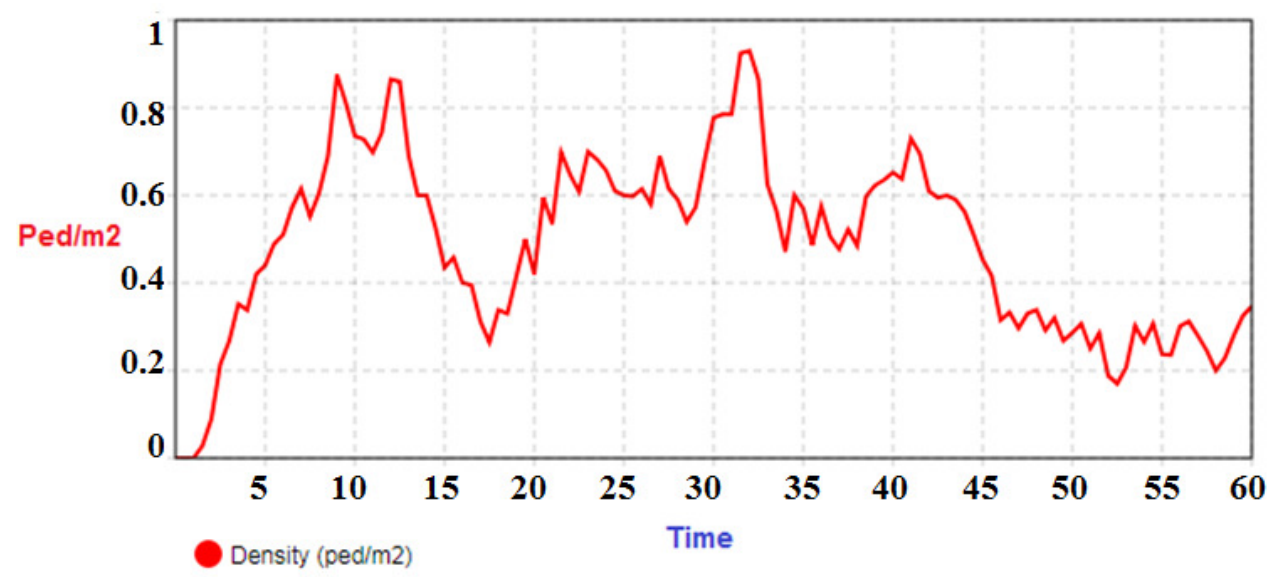

(b)

Figure 11.(a) Density at a specific area without applying the model and (b) when applying the model.

\section{Conclusion And Future Scope}

This model may help in proposing a solution for the congestion problem each year during the Hajj, particularly along the Jamarat path. The image classification results showed that the problem of counting the number of people in the crowd can be solved by using an image classifier that does not depend on counting. The model suggested a solution that does not require telling the pilgrims extensive instructions to follow; they just need to know the meaning of each light. The model uses the idea of traffic lights, with which all people are familiar. Furthermore, the model uses some of the already installed equipment, like cameras.

Using the CNN algorithm has achieved success in experimental evaluations with the available dataset. More work can be done regarding this topic, and for future research, it is recommended to acquire a more suitable dataset; this will require installing new cameras at the proper locations along the Jamarat path to capture good images. To do this, the researchers would need permission from the authorities and a suitable budget for the cameras and the installation process.

We also recommend the use of supercomputers for the computation processes to more easily process the huge number of images, which will result in a more robust classifier. Testing this model in a real environment would also be beneficial for monitoring its efficiency and for making 
further improvements. Finally, we recommend testing the model in locations other than the Jamarat path, such as at events that gather an enormous number of people.

\section{REFERENCES}

[1] Z. Memish, G. Stephens, R. Steffen and Q. Ahmed, "Emergence of medicine for mass gatherings: lessons from the Hajj," The Lancet Infectious Diseases, vol. 12, no. 1, pp. 56-65, 2012.

[2] L. Al-Salhie, M. Al-Zuhair and A. Al-Wabil, "Multimedia surveillance in event detection: crowd analytics in Hajj," Design, User Experience, and Usability. User Experience Design for Diverse Interaction Platforms and Environments, pp. 383-392, 2014.

[3] A. Aljohani, "Pilgrim crowd dynamics," Ph.D., University of Birmingham, 2015.

[4] S. Bandini, S. Manzoni and G. Vizzari, "Agent based modeling and simulation: an informatics perspective," Journal of Artificial Societies and Social Simulation, vol. 12, no. 4, 2009.

[5] I. Khan and R. McLeod, "Managing Hajj crowd complexity: superior throughput,satisfaction,health,\& safety," Arabian Journal of Business and Management Review, vol. 2, no. 4, 2012.

[6] W. Mulyana and T. Gunawan, "Hajj crowd simulation based on intelligent agent," International Conference on Computer and Communication Engineering (ICCCE'10), 2010.

[7] A. Abdelghany, K. Abdelghany, H. Mahmassani and W. Alhalabi, "Modeling framework for optimal evacuation of large-scale crowded pedestrian facilities," European Journal of Operational Research, vol. 237, no. 3, pp. 1105-1118, 2014.

[8] A. Fayoumi, S. Al-Ghoraibi, A. Fadel, F. Al-Aswadi, F. Mujallid and M. Wazzan, "A simulator to improve the pilgrims performance in stoning ritual in Hajj," International Journal of Computer Science and Network Security, vol. 11, no. 5, pp. 141-144, 2011.

[9] Q. Ilyas, "A NetLogo model for RamiAl-Jamarat in Hajj," Journal of Basic and Applied Scientific Research, vol. 3, no. 12, pp. 199-209, 2013.

[10] M. Mohamed, Hajj \&Umrah. Beltsville, MD: Amana Publications, 1996.

[11] A. Krizhevsky, I. Sutskever and G. Hinton, "ImageNet classification with deep convolutional neural networks," Communications of the ACM, vol. 60, no. 6, pp. 84-90, 2017.

[12] J. Gu, Z. Wang, J. Kuen, L. Ma, A. Shahroudy, B. Shuai, T. Liu, X. Wang, G. Wang, J. Cai and T. Chen, "Recent advances in convolutional neural networks," Pattern Recognition, vol. 77, pp. 354-377, 2018.

[13] K. O'Shea, and R. Nash, "An introduction to convolutional neural networks," eprintarXiv, 2015.

[14] D. Stutz, "Understanding convolutional neural networks," Faculty of Mathematics, Computer Science and Natural Sciences, Aachen, 2014.

[15] "Free Video to JPG Converter - make snapshots from video files", Dvdvideosoft.com, 2018. [Online]. Available: https://www.dvdvideosoft.com/products/dvd/Free-Video-to-JPG-Converter.htm. [Accessed: November 1, 2018].

[16] C. Nikolai and G. Madey, "Tools of the trade: a survey of various agent based modeling platforms," Journal of Artificial Societies and Social Simulation, vol. 12, no. 22, 2009.

[17] "AnyLogic: Simulation Modeling Software Tools \& Solutions for Business," Anylogic.com, 2018. [Online]. Available: https://www.anylogic.com. [Accessed: August 1, 2018].

[18] D. Helbing and P. Molnár, "Social force model for pedestrian dynamics," Physical Review E, vol. 51, no. 5, pp. 4282-4286, 1995.

[19] J. Fruin, Pedestrians, 1st ed. Washington, D.C.: Highway Research Board, 1971. 